Rechtsmedizin 2013 - 23:77-78

DOI 10.1007/s00194-013-0878-5

Online publiziert: 27. Februar 2013

c) Springer-Verlag Berlin Heidelberg 2013

\section{F. Ramsthaler ${ }^{1} \cdot$ M.A. Verhoff ${ }^{2}$}

${ }^{1}$ Institut für Rechtsmedizin, Universität des Saarlandes, Homburg/Saar

${ }^{2}$ Institut für Rechtsmedizin, Universität Gießen, Gießen

\title{
Forensische Anthropologie
}

\author{
Komplexe Wissenschaft hinter \\ unscheinbarer Fassade
}

Die unvermindert große mediale Aufmerksamkeit der Disziplin scheint mit der objektiven und der relativen Bedeutsamkeit anthropologischer Fallarbeit innerhalb des rechtsmedizinischen Alltags zu kontrastieren. Nur Wenigen ist geläufig, welche innovative Welt sich im Umfeld der Anthropologie entwickelt hat. Die Anthropologie profitiert als , applied science" von zahlreichen Nachbardisziplinen und ihren technischen Entwicklungen. Das Jahrzehnt der Akkreditierungen und Leitlinien ist nicht spurlos an dem Fach vorbeigezogen. Bemühungen um Standardisierungen und Methodenweiterentwicklungen haben den Blick für die Möglichkeiten und Grenzen der Verfahren geschärft sowie eine Fortentwicklung der Methoden vorangetrieben.

Zur Bestandsaufnahme gehört ein schonungsloser Faktencheck. Die institutionelle Anthropologie hat weitere Einschnitte und Schließungen verkraften müssen, und der Blick in die Zukunft gibt keinen Anlass zu übermäßigem Optimismus.

Auf der anderen Seite finden wir eine Neuorientierung in der Bedarfsanalyse. Sich verändernde Populationen, sei es durch Globalisierung und Migration, sei es durch veränderte Ernährungsgewohnheiten und Lebensweisen führen zu neuen rechtsrelevanten Fragestellungen an die Forensik und zu veränderten Anforderungen an die Methoden des Fachs. Etablierte Methoden verlieren ihre uneingeschränkte Gültigkeit. Moderne Bürgerkriege und Massenkatastrophen hinterlassen Opfer, deren Identifizierung oft ohne forensisch-anthropologisches Expertenwissen nicht gelingen kann. Insofern besteht kein Zweifel daran, dass auch in $\mathrm{Zu}$ kunft Bedarf an Fachwissen und Fortentwicklung der verwendeten Methoden besteht. Es ist eine große Herausforderung an die gegenwärtigen Verantwortlichen, den kurzsichtigen Spartrends und SchlieBungen durch Überzeugung und Argumente ein Gegengewicht entgegenzusetzen. Grundsatzdiskussionen, inwieweit die Rechtsmedizin als medizinisches Fach zur Bewältigung anstehender Aufgaben in der Lage sein kann, sollen an dieser Stelle nicht geführt werden, da die Autoren aus Überzeugung die multidisziplinäre Arbeitsweise favorisieren, bei der Wissen und Fähigkeiten verschiedener Kompetenzen kooperativ zusammengeführt werden.

Die Sonderausgabe der Zeitschrift Rechtsmedizin hat nicht zum Ziel, existierende Monografien und Sammelbände zu ersetzen. Hier soll auf die umfangreiche Literatur und die zahlreichen Übersichtsbeiträge verwiesen werden. Wir haben vielmehr neben einer allgemeinen Bestandsaufnahme gezielt eine Gruppe von Themen ausgewählt, die gegenwärtige Trends und Probleme aufzeigen sollen.

Der Bedarf an Vergleichs- und Untersuchungsmaterial in osteologischen Studien kann nur durch moderne digitale Techniken gedeckt werden. Ousley u. Jantz geben einen ersten Ausblick über denkbare Methoden und Strukturen einer zukünftigen europäischen Datenbank sowie die Weiterentwicklung der bewährten Software „Fordisc“.
Das Bergen von Skelettresten ist ein gelegentlich schwieriges Unterfangen im rechtsmedizinischen Umfeld. Exhumierungsstrategien stoßen schnell an ihre Grenzen. Hier kann die Rechtsmedizin von den umfangreichen Kompetenzen und Erfahrungen sowie den technischen Möglichkeiten der Anthropologie, Archäologie und Geowissenschaften lernen. Gleichzeitig sollen die vorgestellten Arbeiten zu einer vermehrten Zusammenarbeit motivieren, wenngleich sie jedem Leser sofort die pekuniären Grenzen solcher Überlegungen bewusst lassen werden.

Die interessantesten technischen Innovationen, die in die forensische Anthropologie Eingang gefunden haben, stammen zweifelsfrei aus der diagnostischen Medizinforschung. Bildgebung ohne digitale Technik ist heute auch in der forensischen Anthropologie unvorstellbar. Dass der Einsatz von modernen Technologien sich nicht auf radiologische Schnittbildverfahren beschränken muss, wird in einem weiteren Aufsatz vorgestellt.

In Zeiten allgegenwärtiger Sparzwänge sind fundierte Überlegungen zu Aufwand, Umfang und Nutzen wissenschaftlicher Studien wichtiger denn je. Jede durchgeführte Studie steht wie jede neu vorgestellte Methode auf dem Prüfstand ihrer Teststärke, Validität und Reliabilität. Das Sonderheft hat sich daher das Ziel gesetzt, einige der häufig auftretenden statistischen Grundfragen zur Fallzahlplanung zu erörtern.

Wir hoffen, mit diesem Sonderheft der Zeitschrift Rechtsmedizin interessier- 
te Leser ermuntern zu können, sich diesem interessanten Spezialgebiet vermehrt $\mathrm{zu}$ widmen, sich auf interdisziplinäre $\mathrm{Zu}$ sammenarbeit einzulassen und hierdurch auch mitzuhelfen, dem Fach ein zukunftsfähiges Forum zu schaffen.

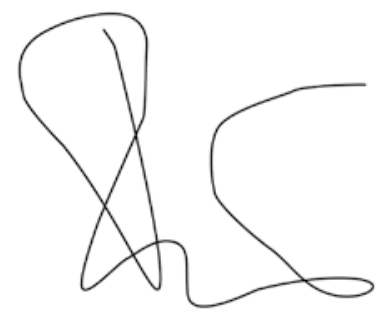

F. Ramsthaler

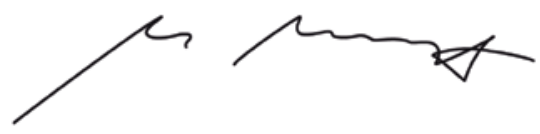

M.A. Verhoff

\section{Korrespondenzadressen}

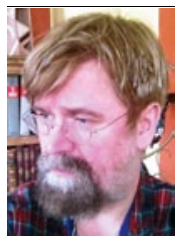

Dr. F. Ramsthaler

Institut für Rechtsmedizin,

Universität des Saarlandes

Gebäude 42,

66421 Homburg/Saar

Frank.Ramsthaler@ uniklinikum-saarland.de

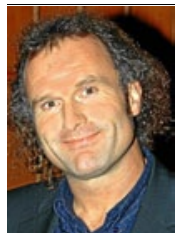

Prof. Dr. M.A. Verhoff

Institut für Rechtsmedizin,

Universität Gießen

Frankfurter Str. 58,

35392 Gießen

Marcel.A.Verhoff@

forens.med.uni-giessen.de

Interessenkonflikt. Die korrespondierenden Autoren geben an, dass kein Interessenkonflikt besteht.

\section{R. Müller-Isberner, S. Eucker (Hrsg.) \\ Praxishandbuch \\ Maßregelvollzug}

Grundlagen, Konzepte und Praxis der

Kriminaltherapie

MWV 2012, 2. Aufl., 311 S., 49.00 EUR

Die Herausgeber nutzen ihre Erfahrungen

in Behandlung, Patientenversorgung und

Organisation des Maßregelvollzugs in Hessen und geben einen fundierten Abriss zur Behandlung straffällig gewordener Patienten. Zusätzlich enthält das Praxishandbuch Beiträge weiterer ebenfalls mit Patienten aus dem Maßregelvollzug in Hessen arbeitender Personen aus verschiedenen Disziplinen. Es entsteht ein abgerundeter und fundierter Überblick über die gesamte Maßregelvollzugsbehandlungskette am Beispiel Hessens. Die 2. Auflage des Praxishandbuchs baut auf das 2009 erschienene „Therapie im Maßregelvollzug" auf und ist verglichen mit dem Vorgängerwerk erweitert, ergänzt und jetzt auch interdisziplinär angelegt. Auf 301 Seiten werden rechtliche Basis, Grundlagen, Methoden und Praxis der Kriminaltherapie, Therapiekonzepte bei verschiedenen Patientengruppen, spezielle Behandlungssettings, die Unterbringung in einer Entziehungsanstalt gemäß § 64 StGB sowie weitere mit dem Maßregelvollzug verbundene Themenfelder aufgegriffen. Es resultiert eine insgesamt gelungene, praxisorientierte und wissenschaftlich fundierte Darstellung der Maßregelvollzugsbehandlung. Eine Stärke des Buches ist, dass auch der für einen Großteil der dort behandelten Patienten zuständige Vorsitzende Richter der Strafvollstreckungskammer beim Landgericht Marburg als Autor einbezogen ist und die juristischen Grundlagen der Arbeit im Maßregelvollzug erörtert.

Man mag den Begriff, Kriminaltherapie“ nicht mögen, schließlich geht es um die Behandlung psychisch kranker Menschen, die aufgrund ihrer Störung straffällig geworden sind, das in diesem Kapitel zusammengetragene empirische Grundlagenwissen und die praxisrelevanten Darstellungen sind uneingeschränkt lesenswert. Nachdem der Maßregelvollzug in Hessen bis vor kurzem organisatorisch zusammengehörig und unter einer Leitung verbunden war, konnten sich die Autoren auf eine landesweite Behandlungsevaluation stützen. Es ist die große Stärke des Buches, dass es auf fundierten
Kenntnissen und praktischem Arbeiten im Maßregelvollzug und mit Maßregelvollzugspatienten basiert. Praktische Erfahrungen mit Evaluation und Erfolgskontrolle fließen in das Praxishandbuch Maßregelvollzug ein und machen es zu einer wertvollen Lektüre für alle im Maßregelvollzug Tätigen. Insofern ist das Buch uneingeschränkt zu empfehlen. Kritisch anzumerken ist nur weniges: Neben meinem Unbehagen mit den Begriffen "Kriminaltherapie" und „Funktionelle Psychosen" (zumindest die älteren wissen noch was gemeint ist) hielte ich die Kapitelbezifferung für verbesserbar. Dies soll den insgesamt sehr positiven Eindruck aber allenfalls marginal schmälern. Etwas gravierender fällt meines Erachtens ins Gewicht, dass Sexualstraftäter und die Diagnose Sexuelle Devianz eher ein Schattendasein führen. Das Wissen hierzu ist im Wesentlichen auf 3 Seiten komprimiert. Dies könnte angesichts der aktuellen Bedeutung dieser Störungen meines Erachtens sicher etwas umfangreicher ausfallen. Insgesamt jedoch haben Herausgeber und Autoren ein wichtiges und sehr hilfreiches Werk zustande gebracht.

J.L. Müller (Göttingen) 